\title{
Les professionnels scolaires: travail, défis et contribution à la vocation démocratique de l'école québécoise
}

\section{HRONIQUE • Profession de l'éducation}

Considérer les enseignants comme étant le seul corps d'emploi professionnel constitue un obstacle à une meilleure connaissance de l'organisation du travail au sein des établissements scolaires. On retrouve effectivement dans nos écoles des professions qui ne se laissent aucunement réduire, stricto sensu, à l'enseignement : psychologues, neuropsychologues, psychoéducateurs, orthophonistes, ergothérapeutes, conseillers d'orientation, animateurs de vie spirituelle et d'engagement communautaire, coordonnateurs de programmes Sports-Arts-Études, conseillers pédagogiques qui relèvent des commissions scolaires et qui encadrent, sur le terrain même, les différents professionnels qui œuvrent dans les établissements. Et on retrouve même des professionnels appartenant à des institutions autres que scolaires comme des travailleurs sociaux, des infirmières et des policiers.

Au cours des 10 dernières années, des chercheurs issus des sciences de l'éducation ou des sciences sociales, comme la sociologie, nous ont fait découvrir le travail de différents agents scolaires, dont les enseignants, les directeurs d'établissement et les techniciens en éducation spécialisée. Les contributions aux prochains numéros de la présente chronique proviennent des professionnels scolaires eux-mêmes. Nous les invitons à nous dire en quoi consistent leurs principales fonctions et responsabilités au sein des écoles primaires ou secondaires, comment ils collaborent avec les enseignants et les autres professionnels, et surtout, si l'organisation des services complémentaires, auxquels ils appartiennent, a tendance actuellement à se redéfinir, et en quoi, le cas échéant, une telle redéfinition serait indicative de profondes mutations quant aux missions fondamentales de l'école québécoise qui dépassent les limites strictes de l'enseignement en classe. 


\section{Les enjeux reliés au travail des professionnels scolaires}

Mais en quoi le travail des professionnels scolaires réalisé avec des élèves aux besoins diversifiés seraitil indicatif de changements importants au sein de l'école québécoise? En fait, il s'agit là d'une question de recherche qui oriente certains des travaux des responsables de cette chronique depuis le début des années 2000 et qui, en soi, est loin d'être prédominante pour les agents scolaires qui se préoccupent moins du sens que prennent les changements au sein des systèmes éducatifs que de la réussite de leurs propres élèves. Deux réflexions illustrent un tel état d'esprit des agents scolaires. La première est celle de la sociologue de l'éducation française, Nathalie Mons, qui a mené des recherches éminemment éclairantes sur les nouvelles politiques scolaires d'inspiration néolibérale axées sur la performance (et l'égalité) de tous les systèmes éducatifs des pays de l'OCDE. Elle mentionne que toutes ces transformations qui touchent à l'organisation des services dans les écoles affectent directement le travail des enseignants, mais qu'elles sont pourtant loin des préoccupations de ceux-ci, ce que semble confirmer cette seconde réflexion qui provient d'une professionnelle scolaire rencontrée en 2018 dans le cadre de nos recherches : «le monde de l'enseignement, c'est très pragmatique ».

Pourquoi alors ne pas s'en tenir à des réflexions qui portent exclusivement sur le travail des enseignants et plus particulièrement sur l'efficacité des méthodes pédagogiques? Trois réponses différentes, mais fortement interreliées, permettent de répondre à cette question : 1) depuis la réforme de l'éducation des années 1960, l'école québécoise se définit officiellement par des politiques de démocratisation; 2) la socialisation, qui constitue une des trois missions de l'école québécoise avec l'instruction et la qualification, ne se limite pas à la réduction de la violence dans les établissements, mais comprend plus largement l'intégration scolaire de tous élèves; et 3) de plus en plus d'agents scolaires non enseignants sont affectés à ce travail d'intégration, donc à l'effort de démocratisation de l'école québécoise.

En quoi le travail des professionnels scolaires, qui s'inscrit dans le prolongement de cet effort de démocratisation de l'école québécoise que nous venons d'évoquer, consiste-t-il plus précisément? Certes, nous savons tous ce qu'est un psychologue ou une orthophoniste, mais le travail qu'ils ont à réaliser dans l'enceinte scolaire est-il le même que celui pour lequel ils ont été formés, que celui qu'ils réaliseraient dans une institution autre que l'école ou encore en clinique privée? En quoi consiste plus spécifiquement leur travail dans un établissement scolaire? Comment les différentes catégories de professionnels scolaires conçoivent-elles leur fonction ou leur rôle à l'intérieur de l'école québécoise? Quelles sont les conditions de travail des professionnels scolaires? Leurs pratiques professionnelles ontelles changé depuis 20 ou 30 années? Quels sont les défis reliés à l'inclusion des élèves handicapés ou ayant des difficultés d'adaptation ou d'apprentissage (EHDAA) en classe régulière ou encore en classe d'adaptation scolaire? Comment les professionnels et les différents agents scolaires se coordonnent-ils afin de soutenir le plus grand nombre d'élèves? Parle-t-on alors de travail commun forcé, obligé, de coordination au sens de juxtaposition des interventions des uns et des autres ou d'une réelle collaboration dans le cadre d'équipes multidisciplinaires? Quels sont alors les facteurs qui favorisent ou entravent le travail collectif des professionnels? Les professionnels accordent-ils plus d'importance à l'évaluation cognitive devant servir au classement des élèves ou aux interventions plus psychoaffectives prenant la forme de relation d'aide? Quel rapport les professionnels entretiennent-ils aux données issues de la recherche? Quels principaux défis ou difficultés rencontrent-ils au quotidien dans leur travail? À quoi attribuent-ils leur efficacité dans leur travail? Comment qualifient-ils les rapports qu'ils entretiennent avec les parents et surtout avec les enseignants? 
Les professionnels scolaires, à qui nous céderons la parole au cours des prochains numéros de cette chronique, ont donc la possibilité de se faire entendre par rapport à l'une ou l'autre de ces questions, ou d'autres questions encore qu'eux seuls peuvent formuler en raison de la connaissance des aspects les plus implicites de leur travail qui souvent échappent aux chercheurs les plus expérimentés. Et au-delà de critiques ou même de revendications professionnelles, leur discours est susceptible de mettre en lumière certains des grands enjeux actuels que l'école québécoise, par leur entremise, parvient à relever. C'est à tout le moins le pari que nous faisons.

\section{Pour citer cet article}

LeVasseur, L. (2019). Les professionnels scolaires : travail, défis et contribution à la vocation démocratique de l'école québécoise. Formation et profession. 27(2), 124-126. http://dx.doi.org/10.18162/fp.2019.a177 